\title{
Motivational Priming Predicts How Noxious Unconditioned Stimuli Influence Affective Reactions to Emotional Pictures
}

\author{
Amy E. Williams ${ }^{1}$, Jamie L. Rhudy ${ }^{2}$ \\ ${ }^{1}$ Department of Psychiatry and Behavioral Neurosciences \& Department of Pediatrics, \\ Loyola University Medical Center, Maywood, USA \\ ${ }^{2}$ Department of Psychology, The University of Tulsa, Tulsa, USA \\ Email: amywilliams@lumc.edu
}

Received July $18^{\text {th }}, 2012$, revised August $20^{\text {th }}, 2012$; accepted September $18^{\text {th }}, 2012$

\begin{abstract}
Motivational priming theory (MPT) and preparedness theory generate competing hypotheses about the impact of an aversive US on responses to an affective foreground. MPT predicts the aversive US will facilitate negative emotional reactions to unpleasant pictures and inhibit positive emotional reactions to pleasant pictures. Preparedness theory predicts an aversive US will increase negative emotional reactions to unpleasant pictures, but will not impact responses to pleasant pictures. The present study $(N=125)$ compared these competing hypotheses by assessing how noxious shocks and non-noxious noises influence responses to emotional pictures. Following each picture, participants rated how the picture made them feel using the Self Assessment Manikin. Results supported MPT—noxious USs, but not non-noxious USs, facilitated negative emotional reactions to unpleasant pictures and inhibited positive emotional reactions to pleasant pictures.
\end{abstract}

Keywords: Affect; Noxious Stimulation; Emotional Picture Ratings; Motivational Priming Theory; Preparedness Theory

\section{Introduction}

Experience of an aversive stimulus can impact emotional reactions to an affective foreground, however, motivational priming theory (MPT) and preparedness theory generate competing hypotheses about the direction of this relationship. Better understanding of the nature of the influence of aversive stimuli on emotional reactions to an affective foreground may have important implications for research design and better understanding of emotional responses during clinical pain.

\section{Motivational Priming Theory (MPT)}

MPT conceptualizes emotion as emanating from two opponent motive systems that provide feedback about the environment (Bradley, Codispoti, Cuthbert, \& Lang, 2001; Lang, 1995). The defensive system is activated by stimuli and contexts that represent a potential or actual threat (e.g., predation, attack, noxious events), and activation of the defensive system promotes protective behaviors (e.g., fight, flight, freezing) and the subjective experience of negative affect. In contrast, the appetitive system is activated by stimuli and contexts that advance survival (e.g., sustenance, procreation), and activation of the appetitive system promotes approach/appetitive behaviors (e.g., consummation, copulation) and the experience of positive affect.

MPT posits that defensive responses should be inhibited by appetitive activation and concurrent positive affect, and facilitated by defensive activation and concurrent negative affect (Lang, 1995). Supporting this, numerous studies have shown that priming the defensive system using unpleasant environmental stimuli results in larger startle reflexes (a defensive response), whereas priming the appetitive system with pleasant environmental stimuli generally results in smaller startle reflexes (e.g., Grillon, Ameli, Merikangas, Woods, \& Davis, 1993; Jansen \& Frijda, 1994; Lang, Bradley, \& Cuthbert, 1990; Vrana, Spence, \& Lang, 1988). By contrast, MPT posits that appetitive responses should be inhibited by defensive activation and concurrent negative affect, and facilitated by appetitive activation and concurrent positive affect (Lang, 1995). While fewer studies have examined the modulation of appetitive responses, there is some existing evidence in humans that they are modulated as predicted by MPT (e.g., Benning, Patrick, \& Lang, 2004).

Several studies have supported MPT; however, in the vast majority the focus has been on the influence of an affectivelycharged perceptual foreground (pictures, sounds, odors, etc.) on an unconditioned defensive reflex (e.g., startle reflex, withdrawal response) to an aversive unconditioned stimulus (US; e.g., noise burst, shock, airpuff) (Hawk \& Cook, 1997; Rhudy, Williams, McCabe, Russell, \& Maynard, 2008; Vrana et al., 1988). In addition, the anticipation of an aversive event (e.g., shock delivery) can also facilitate defensive reflexes to an aversive US (Greenwald, Bradley, Cuthbert, \& Lang, 1998; Grillon, Ameli, Woods, \& Merikangas, 1991), suggesting MPT may provide a framework to explain a greater scope of emotional reactions than what is most commonly studied.

More recently, this line of research has been expanded to assess the influence of an affectively-charged foreground on the subjective reaction to a noxious US, specifically the subjective pain response (De Wied \& Verbaten, 2001; Meagher, Arnau, \& Rhudy, 2001; Rhudy, Williams, McCabe, Nguyen, \& Rambo, 
2005). Consistent with MPT, pain is inhibited in the context of a pleasant foreground and enhanced in the context of an unpleasant foreground. Again, in most of these studies, interest has been on the influence of the affectively-charged foreground on defensive responses to the US.

Less is known about the reverse; the effects of an aversive US on subjective responses to the affective foreground (e.g., ratings of affectively-charged pictures). Some research has indicated that presentation of a painful stimulus does alter participant's emotional state (Bresin, Gordon, Bender, Gordon, \& Joiner, 2010). Presumably if MPT is correct, then an aversive US could influence emotional reactions to affectively-charged pictures. In a study by Bradley and colleagues (Bradley, Codispoti, Cuthbert, et al., 2001), participants viewed emotionallycharged pictures and an aversive, but non-noxious, white noise (50-ms, 96-dB) was delivered during half of the pictures. Immediately following each picture, participants were asked to rate their affective pleasure/valence and arousal response to the picture. They found that noises slightly increased the arousal ratings, but not the pleasure/valence ratings of pictures. While the effects of the aversive US on responses to pictures were minimal in that study, it is not clear whether a more potent (i.e., noxious) US would have a greater impact. Indeed, the degree of motive system activation (as assessed from affective arousal/ intensity) is believed to influence the degree of motivational priming effects (e.g., Bradley, Codispoti, Cuthbert et al., 2001; Cuthbert, Bradley, \& Lang, 1996; Rhudy et al., 2008). Specifically, unpleasant stimuli that elicit higher emotional arousal elicit stronger facilitation of defensive responses, and pleasant stimuli that elicit higher emotional arousal elicit stronger inhibition of defensive responses. For example, our laboratory has demonstrated that erotica (highly arousing pleasant stimuli) inhibits pain and nociceptive reflexes to noxious shocks, but that pictures of food or families (low arousal pleasant stimuli) have little effect on pain and nociceptive reflexes (Rhudy et al., 2010; Rhudy et al., 2008). Thus, MPT would predict that the presentation of a noxious US during an affective foreground would increase (facilitate) negative emotional responses to an unpleasant foreground and decrease (inhibit) positive emotional responses to a pleasant foreground.

\section{Preparedness Theory}

By contrast, the influence of the noxious US on emotional reactions to affectively-charged pictures might be better predicted by preparedness theory (e.g., Peterson, Maier, \& Seligman, 1993; Seligman, 1970, 1971). Research supporting preparedness theory has primarily focused on the influence of an aversive US on responses to an affective foreground. Specifically, preparedness theory posits that unpleasant, fear-relevant stimuli are more readily associated with a noxious US than are pleasant, non-fear-relevant stimuli (Peterson, et al., 1993; Seligman, 1970, 1971). Differences in cognitive mechanisms involved in prepared vs un-prepared learning are thought to be responsible for these differing outcomes (Ohman \& Dimberg, 1978; Ohman \& Soares, 1993). Prepared learning (associations between fear-relevant stimuli and a noxious US) is a non-cognitive learning process thought to be learned through evolution. In contrast, un-prepared learning (associations between nonfear-relevant stimuli and a noxious US) is dependent upon cognitive associations and expectancies. As a result, when a US is paired with an unpleasant stimulus (prepared learning), a condi- tioned response will occur more quickly, will be a stronger response, and will be more resistant to extinction (Ohman \& Dimberg, 1978; Ohman \& Soares, 1993). For example, a recent study found that pairing a noxious electric stimulus (US) with a photograph of a fearful facial expression resulted in greater skin conductance response, lower emotional valence ratings, and higher emotional arousal ratings compared to when the photograph was presented without the US (Williams \& Rhudy, 2007). However, there was not a significant change in physiological or self-reported emotional response to a photograph of a happy facial expression that had been paired with the same US. Thus, preparedness theory predicts a different pattern of results than MPT. Preparedness theory predicts that a noxious US would increase negative emotional responses to an unpleasant picture, but would not alter the emotional response to a pleasant picture (Peterson et al., 1993; Seligman, 1970, 1971).

\section{Present Study}

The purpose of the present study was to examine the influence of a noxious US on emotional reactions to affectivelycharged pictures and determine which of these competing theories best explains the results. This could contribute to a better understanding of how noxious stimuli impact emotional responses to affective foregrounds, which may have implications for research designs and emotional responding in the presence of clinical pain. Data were analyzed from a large study $(N=$ 125 ) involving the presentation of a series of emotional pictures during which noxious electric stimulations were delivered. Following each picture, participants used the Self-Assessment Manikin (SAM) to make ratings of hedonic valence/pleasure and arousal. Consistent with prior research (Rhudy et al., 2005; Rhudy, Williams, McCabe, Rambo, \& Russell, 2006; Rhudy et al., 2008), the results of the parent study found that arousing unpleasant pictures (mutilation) enhanced pain and the nociceptive flexion reflex (NFR, a spinally-mediated withdrawal reflex), whereas arousing pleasant pictures (erotica) inhibited pain and the NFR (Rhudy et al., 2010). The present study represents secondary analyses on this dataset to determine whether the delivery of noxious shock USs influenced emotional reactions to pictures by comparing affective ratings of shocked and unshocked pictures. Because men and women differ in their emotional reactivity to affective pictures (Bradley, Codispoti, Sabatinelli, \& Lang, 2001), we also included participant sex as a factor in all analyses to control for these sex effects. However, sex was not expected to moderate the influence of the US on emotional reactions to pictures.

Additionally, analyses were conducted to examine the impact of aversive, but non-noxious, acoustic startle probes on emotional reactions to pictures. Aversive acoustic startle probes were expected to elicit less defensive activation than noxious shocks, allowing for a comparison of two types of USs that differ primarily in their threat value (degree of defensive system activation). Exposure to noxious shocks can cause sensitization of responses to acoustic startle probes (Greenwald et al., 1998); therefore, testing with acoustic probe USs always preceded testing with shock USs. We hypothesized that noxious USs would lead to enhanced negative emotion when administered during unpleasant images and inhibited positive emotion when administered during pleasant images. In comparison, non-noxious USs were hypothesized to have little to no impact on emotional reactions to pictures. 


\section{Method}

\section{Participants}

Participants were recruited from The University of Tulsa and the Tulsa community through posted announcements on campus and on various community boards in the city of Tulsa. Participants were 18 years of age or older and were excluded for a self-reported history of any condition that may interfere with emotion or pain processing: cardiovascular, neurological, and/ or circulatory problems; use of analgesic, antidepressant, or anti-anxiety medication; recent psychological trauma; specific phobia of snakes or spiders (due to picture-viewing); problems healing; or Raynaud's disease. All procedures were fully approved by The University of Tulsa ethics review board and participants gave verbal and written informed consent after details of the experiment were fully described to them. One hundred fifty one participants completed the study, however, one was excluded for incomplete data and 25 were excluded because they did not experience the shock US as noxious (at least 25 on the 0 - 100 numerical rating scale, see description below). Participants $(N=125)$ were mostly female $(62 \%)$, White-non Hispanic (74\%), single (56\%), and employed (71\%), with an average age of 35 years (range $18-83 ; S D=14.41$ ). Participants received $\$ 100$ for their participation.

\section{Apparatus}

A computer running LabVIEW software (National Instruments, Austin, TX) equipped with dual monitors and A/D board (National Instruments, PCI-6036E) controlled all stimuli, questionnaire presentation, and data acquisition, and was used for offline data reduction. One 17 flat panel monitor was used by the experimenter to monitor physiological signals and experimental timing. Pictures and questionnaires were projected onto a screen about $3 \mathrm{~m}$ from the participant using an LCD projector. Acoustic startle probes were delivered by a Coulbourn Instruments (Allentown, PA) audio signal generator (Part number A12-33) and amplified by a 40W amplifier (Radio Shack, Part \#32-2054). The noise probes were presented over TDH-49 Telephonics (Farmingdale, NY) headphones. Electrocutaneous stimulations were delivered by a Grass Instruments stimulator (Model S88 or S48, West Warwick, RI), stimulus isolation unit (Model SIU8T), constant current unit (Model CCU1), and bipolar stimulating electrode (Nicolet, 019-401400, Madison, WI). The stimulating electrode was attached to the left ankle over the retromalleolar pathway of the sural nerve. The onset/ offset of the stimulator was controlled by computer, and a computer-controlled voltage regulator varied the current to the participant $(\max$ current $=40 \mathrm{~mA}$ ). To apply stimulating electrodes, first the skin was degreased with alcohol, slightly abraded using NuPrep gel to achieve impedances below $5 \mathrm{~K} \Omega$, and then conductive gel (EC60, Grass Instruments) was applied. Throughout testing, participants were seated in a recliner with a small pillow positioned under their left ankle to facilitate relaxation. Sound attenuating headphones and a video camera allowed the experimenter to communicate with and monitor the participant from an adjacent room. Participant ratings were made using a computer mouse positioned on a lap desk.

\section{Picture-Viewing}

Digital pictures from the International Affective Picture Sys- tem (CSEA, 1999; Lang, Bradley, \& Cuthbert, 1999) were presented by computer and an LCD projector in a dimly lit room. During testing with non-noxious acoustic probes, 54 pictures were presented consisting of 18 unpleasant (6 mutilation, 6 attack, 6 death/loss), 18 pleasant ( 6 family, 6 adventure/ sports, 6 erotic) and 18 neutral pictures. ${ }^{1}$ The order of picture presentation was randomized across participants with the limitation that not more than two pictures of similar valence were shown consecutively. During testing with noxious electrocuteneous stimuli, pictures were presented in four blocks of 27 pictures with picture order randomized within each block. There were 36 unpleasant (12 mutilation, 12 attack, 12 death/ loss), 36 pleasant (12 family, 12 adventure/sports, 12 erotic) and 36 neutral pictures for a total of 108 pictures. ${ }^{2}$ Each block contained three pictures from each emotional content and nine neutral pictures. All pictures were presented for $6 \mathrm{~s}$ and startle probes or electric shocks were delivered $3-5 \mathrm{~s}$ following picture onset. Inter-picture intervals varied randomly between 12 and 22 seconds.

\section{Affective Valence and Arousal Ratings}

Affective ratings of each picture were assessed using the Self-Assessment Manikin (SAM; Bradley \& Lang, 1994). The SAM is a two item questionnaire that consists of two sets of five pictographs depicting affective valence/pleasure (unpleasant-pleasant) and arousal (calm-excited). A computerized version of the SAM was used to rate affective valence and arousal. Immediately following each picture the SAM was presented and participants were asked to rate their emotional reaction to the picture (Bradley, Codispoti, Cuthbert et al., 2001; Schupp, Cuthbert, Bradley, \& Birbaumer, 1997). To respond, participants dragged an indicator on or between any of the five picotgraphs for each scale and submitted their answers by computer mouse. This yielded ratings between 1 and 9 for each dimension, with higher scores indicating greater pleasure or arousal for valence and arousal dimensions, respectively.

\section{Unconditioned Stimuli: Non-Noxious Acoustic Probes \& Noxious Electric Stimulations}

Acoustic probes were delivered randomly during $50 \%$ of the pictures $3-5 \mathrm{~s}$ after picture onset, and were equally distributed across picture contents and picture valences. Startle probes were also delivered during $33.3 \%$ of inter-picture intervals, 11 $21 \mathrm{~s}$ after interval onset. Each startle probe consisted of a $50 \mathrm{~ms}$

\footnotetext{
${ }^{1}$ Image numbers during context of acoustic probe presentation were: Erotica $(4607,4608,4660,4677,4800,4810)$, Adventure/Sports $(5450,8170,8191$ $8210,8251,8380)$, Families $(2160,2222,2331,2345,2442,2550)$, Neutral $(2320,5510,7002.7006,7009,7025,7031,7035,7041,7150,7175,7179$, 7211, 7217, 7233, 7236, 7830, 7950), Death/Grieving (2455, 2700, 2900, $4598,6020,9001)$, Attack $(1052,1300,1930,3500,6230,6540)$, Mutilation $(3015,3030,3061,3100,3102,3140)$.

${ }^{2}$ Image numbers during context of shock presentation were: Erotica (4647, $4651,4652,4658,4659,4664,4670,4672,4681,4683,4687,4689)$, Adventure/Sports $(2216,5460,5470,5626,8116,8161,8192,8193,8260$ $8300,8340,8470)$, Families $(2165,2170,2208,2224,2303,2310,2311$, $2340,2341,2395,2655,2660)$, Neutral $(2190,2480.2570,2580,2880$, $5390,5500,5520,2230,5531,5533,5534,6150,7000,7004,7010,7020$, 7030, 7034, 7038, 7040, 7050, 7060, 7080, 7090, 7100, 7110, 7187, 7190, $7205,7224,7234,7235,7705,7820,7900)$, Death/Grieving $(2141,2205$, 2276, 2800, 2810, 9000, 9220, 9421, 9430, 9440, 9480, 9490), Attack $(1050,1120,1321,1525,1931,1932,3530,6250,6260,6350,6510,6550)$, Mutilation $(3051,3062,3063,3064,3068,3110,3150,3168,3250,9252$, 9253, 9405).
} 
burst of white noise delivered over Telephonics headphones at $105 \mathrm{~dB}-\mathrm{A}$.

Noxious electric stimulations were delivered during $33.3 \%$ of the pictures 3 - $5 \mathrm{~s}$ after picture onset. Noxious stimulations were also delivered during $15 \%$ of inter-picture intervals, $11-21 \mathrm{~s}$ after interval onset. Each electrocutaneous stimulus consisted of a train of five 1-ms pulses at $250 \mathrm{~Hz}$ (3-ms ITI). The level of electric stimulation was held constant during the picture viewing phase of the experiment and was set $20 \%$ above a physiologically-determined threshold, i.e., nociceptive flexion reflex (NFR) threshold. The NFR is a spinally-mediated withdrawal reflex elicited by activation of A-delta afferents (pain fibers) (Sandrini et al., 1999). The muscle activity associated with the NFR is quantified from biceps femoris (hamstring) muscle electromyography (France, Rhudy, \& McGlone, 2009; Rhudy $\&$ France, 2007). The stimulus level that reliably elicits the NFR (NFR threshold) correlates highly with subjective pain threshold and requires the activation of pain fibers (Chan \& Dallaire, 1989; Guieu, Blin, Pouget, \& Serratrice, 1992; Sandrini et al., 2005). Thus, setting the stimulus intensity above NFR threshold maximizes the probability that the stimulations will be noxious (i.e., activates pain fibers) and eliminates the potential for report bias. NFR threshold was assessed immediately after testing with the acoustic probe and pictures, but prior to testing with noxious electric stimulations and pictures. For a detailed description of NFR threshold procedures see the parent study (Rhudy et al., 2010).

Numerical rating scale. After each picture during which a noxious stimulation was delivered, the computer presented a vertical numerical rating scale ranging from 0 to 100 with labels of 0 (no sensation), 1 (just noticeable), 25 (uncomfortable), 50 (painful), 75 (very painful), and 100 (maximum tolerable). Participants dragged an indicator to the desired point to rate the painfulness of the noxious stimulation. Participants were included in analyses if their average NRS rating was $\geq 25$.

\section{Procedure}

Upon arrival to the testing session, participants were provided with an overview of the study and informed consent was obtained. A brief health questionnaire and interview were administered to determine eligibility. Eligible participants were then familiarized with the procedures and SAM ratings, and then electrodes were applied. Participants were told that there were several phases to the experiment. First, startle modulation was examined by presenting acoustic startle probes $(50 \mathrm{~ms}, 105$ dB-A) during $50 \%$ of 54 pictures varying in emotional content and $33.3 \%$ of inter-picture intervals.

After testing the acoustic probes, NFR threshold was determined. Finally, four blocks of 27 pictures (108 total) were presented during which electric stimulations set at $120 \%$ NFR threshold (in $\mathrm{mA}$ ) were randomly delivered during 33.3\% of pictures and $15 \%$ of inter-picture intervals. The SAM was administered immediately after each picture in both phases to assess emotional responses to the pictures (Bradley, Codispoti, Cuthbert, et al., 2001; Schupp et al., 1997) and to ensure that any effect of acoustic startle probes and noxious electrocutaneous stimulations on emotional reactions to pictures could be assessed. Ratings from the 54 pictures during startle assessment and the 108 pictures during noxious shock assessment are presented in this paper. When the experiment was completed, participants were thanked and provided an honorarium.

\section{Data Reduction and Analysis}

Valence and arousal ratings were averaged by picture content (mutilation, attack, death/loss, neutral, family, adventure/sports, erotic) and US status (US presented vs US not presented). To determine the influence of noxious shock US vs non-noxious noise US administration on picture ratings, repeated measures analysis of variance (ANOVA) were conducted separately for valence and arousal ratings during noxious shock assessment and noise probe assessments. Picture Content (mutilation, attack, death/loss, neutral, family, adventure/sports, erotica) and US Status (US vs no US) were entered as within-subjects variables and participant sex was entered as a between-subjects variable (given that sex is known to influence affective ratings; Bradley, Codispoti, Sabatinelli et al., 2001). If MPT is correct, then a main effect of US Status for valence ratings should be significant. However, if preparedness theory is correct, then a US Status X Picture Content interaction for valence ratings will be significant.

Bonferroni adjusted means were conducted for follow-up contrasts to control for Type I error rate (reported $p$-values reflect probabilities after Bonferroni correction). GreenhouseGeisser corrections were used to overcome sphericity violations and epsilon $(\varepsilon)$ values are reported following $p$-values. Partial eta-squared $\left(\eta_{p}^{2}\right)$ was used as the effect size for $F$ tests.

\section{Results}

Tables 1 and 2 report valence and arousal ratings and mean normative ratings for each picture content during noxious shock assessment and startle assessment, respectively.

\section{The Influence of Noxious Electric Stimuli on Emotional Reactions to Pictures}

Valence/pleasure ratings. The main effect of picture content was significant for valence/pleasure ratings, $F(6,738)=$ $508.60, p<.001, \varepsilon=.41, \eta_{p}^{2}=.81$. Valence/pleasure ratings of pictures had the following relations; mutilation $<$ (attack and death) $<$ neutral $<$ (erotic and adventure/sports) $<$ family ( $p$ s $<.01)$. There was a significant main effect of participant sex $\left[F(1,123)=3.90, p=.05, \eta_{p}^{2}=.03\right]$ with male participants $(M$ $=4.71, S D=0.07)$ generally rating pictures with higher valence/pleasure than female participants $(M=4.54, S D=0.06)$ regardless of picture content or US status. These effects were qualified by a significant Picture Content $X$ Sex interaction, $F(6$, $738)=6.41, p=.001, \varepsilon=.78, \eta_{p}^{2}=.05$. Male participants provided higher valence ratings than female participants for erotic pictures, and lower valence ratings for family pictures ( $p s$ $<.01)$.

The main effect of US status was significant for valence/ pleasure ratings, $F(1,123)=10.75, p=.001, \eta_{p}^{2}=.08$. Valence/pleasure ratings were lower for shocked pictures $(M=$ $4.58, S D=.05)$ compared to unshocked pictures $(M=4.67, S D$ $=0.05)$. The Picture Content X US Status interaction, the US Status X Sex interaction, and the 3-way interaction were not significant (all $p s>.20$, all $\eta_{p}^{2} s<.02$ ). Notably, the effect size for the main effect of US Status was 4 times the size of the effect size for the US Status x Picture Content interaction.

Arousal ratings. The main effect of picture content was significant for arousal ratings, $F(6,738)=156.91, p<.001, \varepsilon$ $=.61, \eta_{p}^{2}=.56$. Arousal ratings of pictures had the following relations; neutral $<$ family $<$ death $<$ adventure/sports $<$ (erotic, 


\section{A. E. WILLIAMS, J. L. RHUDY}

Table 1.

Affective valence and arousal ratings during noxious shock assessment separated by shock status and participant sex.

\begin{tabular}{|c|c|c|c|c|c|c|c|c|c|c|c|c|c|c|c|}
\hline & & \multicolumn{2}{|c|}{ Mutilation } & \multicolumn{2}{|c|}{ Attack } & \multicolumn{2}{|c|}{ Death/Grief } & \multicolumn{2}{|c|}{ Neutral } & \multicolumn{2}{|c|}{ Family } & \multicolumn{2}{|c|}{ Adv/Sports } & \multicolumn{2}{|c|}{ Erotica } \\
\hline & & $M$ & $S D$ & $M$ & $S D$ & $M$ & $S D$ & $M$ & $S D$ & $M$ & $S D$ & $M$ & $S D$ & $M$ & $S D$ \\
\hline \multicolumn{16}{|c|}{$\begin{array}{l}\text { Valence/Pleasure } \\
\text { Ratings (1 - 9) }\end{array}$} \\
\hline \multirow{2}{*}{ Men } & Shocked & 1.89 & 1.02 & 2.97 & 1.23 & 2.84 & 1.06 & 5.17 & 0.79 & 6.72 & 1.34 & 6.58 & 1.06 & 6.97 & 1.40 \\
\hline & Unshocked & 2.12 & 0.99 & 3.29 & 1.29 & 3.01 & 0.97 & 5.21 & 0.78 & 6.89 & 1.16 & 6.59 & 1.01 & 7.03 & 1.39 \\
\hline \multirow{2}{*}{ Women } & Shocked & 1.74 & 0.97 & 2.58 & 1.33 & 2.58 & 1.15 & 5.14 & 0.88 & 7.35 & 1.14 & 6.47 & 1.14 & 5.96 & 1.73 \\
\hline & Unshocked & 1.77 & 0.87 & 2.82 & 1.22 & 2.65 & 0.93 & 5.24 & 0.79 & 7.42 & 1.09 & 6.40 & 1.09 & 5.98 & 1.80 \\
\hline \multirow{2}{*}{ Totals } & Shocked & 1.81 & 0.99 & 2.74 & 1.30 & 2.69 & 1.02 & 5.15 & 0.84 & 7.09 & 1.26 & 6.52 & 1.10 & 6.37 & 1.67 \\
\hline & Unshocked & 1.91 & 0.94 & 3.01 & 1.27 & 2.80 & 0.96 & 5.23 & 0.78 & 7.20 & 1.14 & 6.48 & 1.06 & 6.41 & 1.72 \\
\hline \multicolumn{2}{|c|}{ Normative Valence Ratings } & 1.93 & 1.32 & 3.02 & 1.78 & 3.05 & 1.72 & 5.06 & 1.23 & 7.33 & 1.60 & 6.92 & 1.60 & 6.66 & 1.88 \\
\hline \multicolumn{16}{|c|}{ Arousal Ratings (1 - 9) } \\
\hline \multirow{2}{*}{ Men } & Shocked & 6.09 & 1.87 & 5.93 & 1.92 & 4.58 & 1.66 & 3.11 & 1.65 & 3.81 & 1.71 & 5.27 & 1.74 & 6.20 & 2.02 \\
\hline & Unshocked & 5.81 & 1.87 & 5.79 & 1.89 & 4.21 & 1.54 & 2.94 & 1.59 & 3.76 & 1.87 & 5.47 & 1.83 & 6.16 & 2.13 \\
\hline \multirow{2}{*}{ Women } & Shocked & 6.04 & 2.05 & 5.87 & 2.13 & 4.49 & 1.61 & 2.89 & 1.52 & 3.46 & 1.70 & 4.92 & 2.01 & 5.57 & 2.36 \\
\hline & Unshocked & 5.93 & 2.03 & 5.73 & 2.06 & 4.32 & 1.63 & 2.72 & 1.48 & 3.47 & 1.86 & 4.98 & 1.91 & 5.51 & 2.17 \\
\hline \multirow{2}{*}{ Totals } & Shocked & 6.06 & 1.97 & 5.89 & 2.04 & 4.53 & 1.63 & 2.98 & 1.57 & 3.60 & 1.71 & 5.06 & 1.91 & 5.82 & 2.24 \\
\hline & Unshocked & 5.88 & 1.96 & 5.75 & 1.99 & 4.28 & 1.59 & 2.81 & 1.53 & 3.59 & 1.73 & 5.18 & 1.91 & 5.78 & 2.17 \\
\hline \multicolumn{2}{|c|}{ Normative Arousal Ratings } & 6.23 & 2.37 & 6.82 & 2.04 & 4.85 & 2.12 & 2.82 & 1.92 & 4.62 & 2.27 & 5.99 & 2.23 & 6.54 & 2.02 \\
\hline
\end{tabular}

Note: Normative ratings were taken from the IAPS manual (Lang, Bradley, \& Cuthbert, 2005). Adv/Sports = Adventure/Sport.

Table 2.

Affective valence and arousal ratings during acoustic probe assessment separated by probe status and participant sex.

\begin{tabular}{|c|c|c|c|c|c|c|c|c|c|c|c|c|c|c|c|}
\hline & & \multicolumn{2}{|c|}{ Mutilation } & \multicolumn{2}{|c|}{ Attack } & \multicolumn{2}{|c|}{ Death/Grief } & \multicolumn{2}{|c|}{ Neutral } & \multicolumn{2}{|c|}{ Family } & \multicolumn{2}{|c|}{ Adv/Sports } & \multicolumn{2}{|c|}{ Erotica } \\
\hline & & $M$ & $S D$ & $M$ & $S D$ & $M$ & $S D$ & $M$ & $S D$ & $M$ & $S D$ & $M$ & $S D$ & $M$ & $S D$ \\
\hline \multicolumn{16}{|c|}{$\begin{array}{c}\text { Valence/Pleasure } \\
\text { Ratings }(1-9)\end{array}$} \\
\hline \multirow{2}{*}{ Men } & Probed & 1.94 & 1.20 & 3.00 & 1.25 & 3.69 & 1.05 & 5.18 & 0.47 & 6.53 & 1.00 & 6.85 & 0.90 & 6.78 & 1.28 \\
\hline & Unprobed & 1.93 & 1.06 & 3.08 & 1.26 & 3.69 & 0.95 & 5.16 & 0.35 & 6.63 & 1.05 & 6.67 & 1.03 & 6.97 & 1.11 \\
\hline \multirow{2}{*}{ Women } & Probed & 1.80 & 1.03 & 2.61 & 1.22 & 3.34 & 1.31 & 5.31 & 0.75 & 7.25 & 1.21 & 6.52 & 1.08 & 5.98 & 1.44 \\
\hline & Unprobed & 1.83 & 1.06 & 2.62 & 1.25 & 3.44 & 1.22 & 5.30 & 0.68 & 7.16 & 1.19 & 6.71 & 1.27 & 6.09 & 1.54 \\
\hline \multirow{2}{*}{ Totals } & Probed & 1.85 & 1.09 & 2.76 & 1.24 & 3.47 & 1.22 & 5.26 & 0.65 & 6.97 & 1.18 & 6.65 & 1.02 & 6.29 & 1.43 \\
\hline & Unprobed & 1.87 & 1.06 & 2.80 & 1.27 & 3.54 & 1.12 & 5.24 & 0.58 & 6.95 & 1.16 & 6.69 & 1.18 & 6.43 & 1.45 \\
\hline \multicolumn{2}{|c|}{ Normative Valence Ratings } & 1.76 & 1.25 & 2.94 & 1.67 & 3.57 & 1.92 & 5.02 & 1.19 & 7.21 & 1.58 & 6.99 & 1.54 & 6.85 & 1.80 \\
\hline \multicolumn{16}{|c|}{ Arousal Ratings (1 - 9) } \\
\hline \multirow{2}{*}{ Men } & Probed & 5.65 & 1.82 & 5.92 & 2.04 & 4.18 & 1.69 & 2.64 & 1.23 & 2.76 & 1.27 & 5.47 & 1.97 & 5.99 & 2.06 \\
\hline & Unprobed & 5.64 & 1.81 & 5.78 & 2.06 & 3.88 & 1.61 & 2.43 & 1.36 & 3.04 & 1.47 & 5.33 & 1.98 & 6.13 & 1.95 \\
\hline \multirow{2}{*}{ Women } & Probed & 5.89 & 1.88 & 6.04 & 1.97 & 4.11 & 1.56 & 2.58 & 1.36 & 2.97 & 1.61 & 4.85 & 1.87 & 5.50 & 1.88 \\
\hline & Unprobed & 5.82 & 1.85 & 6.05 & 1.96 & 4.04 & 1.54 & 2.36 & 1.23 & 2.96 & 1.58 & 5.17 & 2.09 & 5.45 & 1.99 \\
\hline \multirow{2}{*}{ Totals } & Probed & 5.80 & 1.85 & 5.99 & 1.99 & 4.14 & 1.61 & 2.60 & 1.31 & 2.89 & 1.49 & 5.09 & 1.93 & 5.69 & 1.96 \\
\hline & Unprobed & 5.75 & 1.83 & 5.95 & 2.00 & 3.98 & 1.56 & 2.39 & 1.27 & 2.99 & 1.54 & 5.23 & 2.04 & 5.71 & 1.99 \\
\hline \multicolumn{2}{|c|}{ Normative Arousal Ratings } & 6.23 & 2.40 & 6.82 & 2.08 & 4.85 & 2.16 & 2.83 & 1.90 & 4.61 & 2.25 & 5.98 & 2.23 & 6.55 & 2.00 \\
\hline
\end{tabular}

Note: Normative ratings were taken from the IAPS manual (Lang et al., 2005). Adv/Sports $=$ Adventure/Sport. 
attack, and mutilation) ( $p$ s $<.001)$. There were no differences in arousal for erotic, mutilation, and attack pictures $(p s=1.00)$. The main effect of US status was significant for arousal ratings $\left[F(1,123)=12.75, p=.001, \eta_{p}^{2}=.09\right]$ with higher arousal ratings for shocked $(M=5.01, S D=0.13)$ compared to unshocked pictures $(M=4.88, S D=0.14)$. All other effects were non-significant (all $\mathrm{ps}>.05$, all $\eta_{p}^{2} \mathrm{~s}<.02$ ).

\section{The Influence of Non-Noxious Acoustic Probes on Emotional Reactions to Pictures}

Valence/pleasure ratings. The main effect of picture content was significant for valence/pleasure ratings, $F(6,738)=$ $511.48, p<.001, \varepsilon=.45, \eta_{p}^{2}=.81$. Valence/pleasure ratings of pictures had the following relations; mutilation $<$ attack $<$ death $<$ neutral $<$ (family and adventure/sports) $(p s<.02)$. Valence ratings of family pictures were significantly lower compared to erotic pictures $(p<.001)$ which did not differ from adventure/ sports picture ratings $(p=.44)$. This effect was qualified by a significant Picture Content X Sex interaction, $F(6,738)=6.42$, $p=.001, \varepsilon=.76, \eta_{p}^{2}=.05$. Male participants provided higher valence ratings than female participants for attack and erotic pictures, and lower valence ratings for family pictures ( $p s$ $<.05)$. The main effect of US status and the interaction effects were not significant (all $p \mathrm{~s}>.28$, all $\eta_{p}^{2} \mathrm{~s}<.01$ ).

Arousal ratings. The main effect of picture content was significant for arousal ratings, $F(6,738)=237.83, p<.001, \varepsilon$ $=.61, \eta_{p}^{2}=.66$. Arousal ratings of pictures had the following relations; neutral $<$ family $<$ death $<$ adventure/sports $<$ (erotic, attack, and mutilation) $(p s<.01)$. There were no differences in arousal for erotic, attack, and mutilation ( $p s>.57)$. This effect was qualified by a significant Picture Content $X$ Sex interaction, $F(6,738)=2.63, p=.04, \varepsilon=.87, \eta_{p}^{2}=.02$. However, followup comparisons revealed no significant differences between male and female ratings of pictures after Bonferroni corrections ( $p s>.09)$. The main effect of US status and the US status interactions were not significant (all $p \mathrm{~s}>.14$, all $\eta_{p}^{2} \mathrm{~s}<.02$ ).

\section{Discussion}

The present study sought to determine whether motivational priming theory (MPT) or preparedness theory best explained the influence of a noxious shock on emotional reactions to affectively-charged pictures. MPT predicts an increase (facilitation) in negative emotional responses to an unpleasant foreground and decrease (inhibition) in positive emotional responses to a pleasant foreground (Bradley, Codispoti, Cuthbert, et al., 2001). In contrast, preparedness theory predicts an increase in negative emotional responses to an unpleasant picture, but no change in the emotional response to a pleasant picture (Peterson et al., 1993; Seligman, 1970, 1971). Data were analyzed from a study involving the presentation of a series of emotional pictures (mutilation, attack, death/loss, neutral, family, adventure/sports, and erotic) during which noxious shocks were delivered during $33 \%$ of the pictures. Following each picture, participants used the Self Assessment Manikin (SAM) to rate how the picture made them feel.

In general, subjective ratings of emotional valence and arousal were consistent with picture content (i.e. negative pictures having lower valence than positive pictures; positive and negative pictures more arousing than neutral). Results indicated that valence/pleasure ratings were lower (more displeasure) and arousal ratings were higher for pictures (regardless of picture content) during which participants received a shock US compared to pictures without a shock US. This suggests that presentation of the noxious US led to enhanced negative emotional responses to unpleasant pictures and inhibited positive emotional responses to pleasant pictures, which is consistent with MPT.

A similar experimental protocol was used to assess the impact of a non-noxious US (acoustic startle probe) on emotional reactions to affectively-charged pictures. During this procedure, pictures also elicited the expected emotional responses from participants (i.e. negative pictures having lower valence than positive pictures; positive and negative pictures more arousing than neutral). In contrast to results from noxious shock USs, the presence of an aversive, but non-noxious, acoustic probe did not influence reactions to the pictures.

The results of this study contribute to the generalization of, and research support for, our theoretical understanding of how noxious events/stimuli can impact emotional responses. Emotion has been proposed to emanate from two opponent motive systems, defensive and appetitive (Bradley, Codispoti, Cuthbert et al., 2001). According to MPT, activation of the defensive system should facilitate defensive responses and inhibit appetitive responses; whereas activation of the appetitive system should facilitate appetitive responses and inhibit defensive responses. Research supports these hypotheses, but has generally focused on the influence of an affective foreground (e.g., picture presentation, odors) on defensive responses to an aversive unconditioned stimulus (US; shock, loud noise).

In contrast, the present study sought to assess the effects of an aversive and noxious US on responses to an affective foreground. MPT would predict that priming the defensive system by the aversive US would lead to enhanced defensive responses and inhibited appetitive responses, including subjective experience. In comparison, preparedness theory would predict that the aversive US would increase negative affect in response to unpleasant pictures but would have no effect on emotional response to pleasant pictures. Results supported MPT; noxious shock USs enhanced displeasure to unpleasant pictures and reduced pleasure to pleasant pictures, relative to unshocked pictures. In addition, subjective arousal ratings were enhanced during shocked pictures compared to unshocked pictures. This indicates an individual may have a different emotional response to an affective foreground when a noxious US is presented.

As predicted by MPT, these data suggest the presence of a noxious US will impact reactions to unpleasant and pleasant affective foregrounds. Nonetheless, it should be noted that the means in Table 1 suggest a possible trend towards a US Status $\times$ Picture Content interaction with noxious USs having a slightly larger impact on ratings of unpleasant compared to pleasant pictures. This begs the question of whether a significant interaction would have been found with a larger sample, thus providing support for preparedness theory. However, the difference in magnitude of the main effect of US Status and the US Status x Picture Content interaction suggests the main effect, and thus MPT, represents a stronger and more relevant influence on emotional ratings. Indeed, the US Status $\times$ Picture Content interaction (which tests preparedness theory) was a small effect that accounted for only $2 \%$ of the variance in valence ratings. By contrast, the effect size for the main effect of US Status (which tests the MPT hypothesis) was four times larger and explained $8 \%$ of the variance in valence ratings. As a 
result, this helps bolster confidence in the conclusion that MPT predicts the effect of a noxious US on valence reactions to pictures.

In contrast to the effect of noxious stimuli on ratings of unpleasant and pleasant affective foregrounds, when the experiment involved an aversive but non-noxious US (white noise) the presence of the US during a picture did not influence emotional reactions to the affective foreground. This suggests that the defensive activation in response to non-noxious acoustic probes was not strong enough to impact reactions to the foreground. This is also consistent with MPT which predicts that greater levels of defensive activation are elicited by more threatening or emotionally arousing stimuli, such as a noxious shock, compared to less threatening stimuli such as white noise. Increased defensive activation would then lead to greater facilitation of defensive responses and greater reduction of appetitive responses as seen in the present study.

The lack of impact of white noise on ratings of the affective foreground in the present study is similar to the results of Bradley, Codispoti, Cuthbert, \& Lang (2001) who also found no influence of white noise on valence/pleasure ratings of an affective foreground. In contrast to the present results, they did find a small increase in arousal ratings of pictures during which white noise was presented. Inspection of the current data also indicates a very small increase in arousal ratings for pictures with acoustic USs $(M=4.61)$ versus those without USs $(M=$ $4.58)$, but this effect was not statistically significant $(p=.41$, $\eta_{p}^{2}=.01$ ). Thus, both studies suggest that non-noxious acoustic probes have minimal effect on affective reactions to the foreground.

Although not a main goal of the study, our results confirm prior reports of sex differences in emotional reactions to pictures. Specifically, men typically have greater appetitive activetion to erotica and women typically have greater defensive activation to most unpleasant pictures (Bradley, Codispoti, Sabatinelli et al., 2001). In the present study, men had higher valence/pleasure to erotic pictures and women had higher valence/pleasure ratings for family pictures. Additionally in the series of pictures during which startle probes were presented, women had lower valence ratings of attack pictures. Thus, the present study shows a similar pattern of sex differences in valence ratings of affective pictures as seen in previous studies. However, there were no sex differences associated with the influence of the noxious US on emotional responses to pictures, indicating that the presence of a noxious US had the same effect on ratings of emotional pictures for males and females.

\section{Implications}

Results of this study have implications for research design. As evidenced by these data, presentation of a noxious US alters participant reactions to the affective foreground. While this effect resulted in only minor changes in affective ratings, this could nonetheless alter the efficacy of experimental factors meant to manipulate participant affect. Thus, it is important that studies including a noxious US employ adequate manipulation checks to ensure participant's responses to affective foregrounds are not significantly altered by US presentation. Further, this effect on the affective foreground could present a confound in studies attempting to make comparisons to normative data or to groups that do not receive the same noxious US. Monitoring the effect of non-noxious USs is warranted as well, but may be less likely to alter reactions to affective foregrounds.

The present study makes several contributions to the current research literature. First, the study examined the influence of presentation of a noxious stimulus on ratings of an affective foreground, which although similar, is the reverse of most studies which look at the influence of an affective foreground on response to a noxious stimulus. Studies that have looked at similar outcomes have found mixed results. Bresin and colleagues (2010) found that ratings of both positive and negative affect decreased following an experimental pain stimulus, however, these ratings did not refer to a specific foreground. Hollin and Derbyshire (2009) found that cold pressor pain decreased ratings of fear during concurrent presentation of a spider. The present study extended these studies to assess the influence of noxious and non-noxious stimuli on emotional ratings of affective foregrounds (with multiple valence and arousal levels), and results have implications for research (discussed above). Moreover, there may also be clinical implications. The present study suggests that persons experiencing pain may experience less positive emotion in response to a pleasant event, and greater negative emotion in response to an unpleasant event, compared to a person not experiencing pain. This could partly explain the relationship between chronic pain and depression (Kroenke et al., 2011), because pain may promote anhedonia (low positive affect) and enhanced negative affect.

\section{Limitations}

One factor of note in the present study is that pictures differed not only due to the presence, or lack thereof, of a noxious electric stimulation; but shocked pictures also were followed by a numerical rating scale (NRS) to rate the intensity of the stimulation. It is possible that the presence of the NRS altered the context in such a way as to bias participant ratings of picture-evoked emotional responses on the Self-Assessment Manikin (SAM). However, the motivational relevance of a noxious electric stimulation should be much greater than that of the NRS, and thus the contextual salience of the electric stimulation should overshadow that of the NRS. Moreover, the NRS and SAM were presented simultaneously following pictures with concurrent electrical stimulation. Participants were free to choose which scale to complete first and informal observations made by experimenters indicated there was significant variability in the order in which participants completed the NRS and SAM. Therefore, a subset of participants rated emotional responses to pictures prior to rating the electrical stimulation. This may have reduced any potential confound of the NRS on SAM ratings. Nonetheless, future studies should seek to replicate these data with experimental designs that explicitly control for potential contextual confounds, although presenting the NRS in the absence of an electric stimulus might confuse participants and create additional problems.

Another limitation of this study was that it only assessed the impact of aversive USs. MPT would predict that appetitive USs would also effect reactions to affective foregrounds (facilitating appetitive reactions and inhibiting defensive reactions). Thus, future research is needed to confirm this hypothesis.

\section{Summary}

The present study compared competing hypotheses based 
upon MPT and preparedness theory to determine which would better predict the impact of a noxious US on emotional reactions to affectively-charged pictures. These data supported MPT indicating that the presentation of a noxious US (but not a non-noxious US) can influence reactions to the affective foreground by facilitating negative emotional reactions to unpleasant pictures and inhibiting positive emotional reactions to pleasant pictures. Prior research supporting MPT has found that the presentation of an affective foreground influences physiological and emotional reactions to a noxious US. However, the present study is the first to our knowledge to find the reverse. This study highlights importance of understanding the potential impact of aversive stimuli on emotional reactivity in research and clinical settings in which an aversive stimulus is administered.

\section{REFERENCES}

Benning, S. D., Patrick, C. J., \& Lang, A. R. (2004). Emotional modulation of the post-auricular reflex. Psychophysiology, 41, 426-432. doi:10.1111/j.1469-8986.00160.x

Bradley, M. M., Codispoti, M., Cuthbert, B. N., \& Lang, P. J. (2001). Emotion and motivation I: Defensive and appetitive reactions in picture processing. Emotion, 1, 276-298. doi:10.1037/1528-3542.1.3.276

Bradley, M. M., Codispoti, M., Sabatinelli, D., \& Lang, P. J. (2001). Emotion and motivation II: Sex differences in picture processing. Emotion, 1, 300-319. doi:10.1037/1528-3542.1.3.300

Bradley, M. M., \& Lang, P. J. (1994). Measuring emotion: The Self-Assessment Manikin and the semantic differential. Journal of Behavior Therapy and Experimental Psychiatry, 25, 49-59. doi:10.1016/0005-7916(94)90063-9

Bresin, K., Gordon, K. H., Bender, T. W., Gordon, L. J., \& Joiner, T. E. (2010). NO pain, no change: Reductions in prior negative affect following physical pain. Motivation \& Emotion, 34, 280-287. doi:10.1007/s11031-010-9168-7

Chan, C. W., \& Dallaire, M. (1989). Subjective pain sensation is linearly correlated with the flexion reflex in man. Brain Research, 479, 145-150. doi:10.1016/0006-8993(89)91344-9

CSEA (1999). The international affective picture system: Digitized photographs. Gainesville, FL: University of Florida, The Center for Research in Psychophysiology.

Cuthbert, B. N., Bradley, M. M., \& Lang, P. J. (1996). Probing picture perception: Activation and emotion. Psychophysiology, 33, 103-111. doi:10.1111/j.1469-8986.1996.tb02114.x

De Wied, M., \& Verbaten, M. N. (2001). Affective pictures processing, attention, and pain tolerance. Pain, 90, 163-172.

doi:10.1016/S0304-3959(00)00400-0

France, C. R., Rhudy, J. L., \& McGlone, S. (2009). Using normalized EMG to define the Nociceptive Flexion Reflex (NFR) threshold: Further evaluation of standardized scoring criteria. Pain, 145, 211 218. doi:10.1016/j.pain.2009.06.022

Greenwald, M. K., Bradley, M. M., Cuthbert, B. N., \& Lang, P. J. (1998). Startle potentiation: Shock sensitization, aversive learning, and affective picture modulation. Behavioral Neuroscience, 112, 1069-1079. doi:10.1037/0735-7044.112.5.1069

Grillon, C., Ameli, R., Merikangas, K., Woods, S. W., \& Davis, M. (1993). Measuring the time course of anticipatory anxiety using the fear-potentiated startle reflex. Psychophysiology, 30, 340-346. doi:10.1111/j.1469-8986.1993.tb02055.x

Grillon, C., Ameli, R., Woods, S. W., \& Merikangas, K. (1991). Fear-potentiated startle in humans: Effects of anticipatory anxiety on the acoustic blink reflex. Psychophysiology, 28, 588. doi:10.1111/j.1469-8986.1991.tb01999.x

Guieu, R., Blin, P., Pouget, J., \& Serratrice, G. (1992). High level sportsmen and nociceptive flexion reflex of the lower limb. Canadian Journal of Neuroscience, 19, 69-71.

Hawk, L. W., \& Cook III, E. W. (1997). Affective modulation of tactile startle. Psychophysiology, 34, 23.

doi:10.1111/j.1469-8986.1997.tb02412.x

Hollin, G. J. S., \& Derbyshire, S. W. G. (2009). Cold pressor pain reduces phobic fear but fear does not reduce pain. The Journal of Pain, 10, 1058-1064. doi:10.1016/j.jpain.2009.03.015

Jansen, D. M., \& Frijda, N. H. (1994). Modulation of the acoustic startle response by film-induced fear and sexual arousal. Psychophysiology, 31, 565-571. doi:10.1111/j.1469-8986.1994.tb02349.x

Kroenke, K., Wu, J., Bair, M. J., Krebs, E. E., Damush, T. M., \& Tu, W. (2011). Reciprocal relationship between pain and depression: A 12-month longitudinal analysis in primary care. Journal of Pain, 12, 964-973. doi:10.1016/j.jpain.2011.03.003

Lang, P. J. (1995). The emotion probe: Studies of motivation and attention. American Psychologist, 50, 372-385. doi:10.1037/0003-066X.50.5.372

Lang, P. J., Bradley, M. M., \& Cuthbert, B. (2005). The International Affective Picture System (IAPS): Affective ratings of pictures and instruction manual. Technical Report A-6. Gainesville, FL: University of Florida.

Lang, P. J., Bradley, M. M., \& Cuthbert, B. N. (1990). Emotion, attention, and the startle reflex. Psychological Review, 97, 377-395. doi: 10.1037/0033-295X.97.3.377

Lang, P. J., Bradley, M. M., \& Cuthbert, B. N. (1999). International affective picture system (IAPS): Instruction manual and affective ratings. Technical Report A-4. Gainesville, FL: The Center for Research in Psychophysiology, University of Florida.

Meagher, M. W., Arnau, R. C., \& Rhudy, J. L. (2001). Pain and emotion: Effects of affective picture modulation. Psychosomatic Medicine, 63, 79-90.

Ohman, A., \& Dimberg, U. (1978). Facial expressions as conditioned stimuli for electrodermal responses: A case of "preparedness"? Journal of Personality \& Social Psychology, 36, 1251-1258. doi: $10.1037 / 0022-3514.36 .11 .1251$

Ohman, A., \& Soares, J. J. F. (1993). On the automatic nature of phobic fear: Conditioned electrodermal responses to masked fear-relevant stimuli. Journal of Abnormal Psychology, 102, 121-132. doi:10.1037/0021-843X.102.1.121

Peterson, C., Maier, S. F., \& Seligman, M. E. P. (1993). Learned Helplessness: A theory for age of personal control. New York, NY: Oxford University Press.

Rhudy, J. L., Bartley, E. J., Williams, A. E., McCabe, K. M., Chandler, M. C., Russell, J. L. et al. (2010). Are there sex differences in affective modulation of spinal nociception and pain? The Journal of Pain, 11, 1429-1441. doi:10.1016/j.jpain.2010.04.003

Rhudy, J. L., \& France, C. R. (2007). Defining the nociceptive flexion reflex (NFR) threshold in human participants: A comparison of different scoring criteria. Pain, 128, 244-253. doi:10.1016/j.pain.2006.09.024

Rhudy, J. L., Williams, A. E., McCabe, K. M., Nguyen, M. A., \& Rambo, P. L. (2005). Affective modulation of nociception at spinal and supraspinal levels. Psychophysiology, 42, 579-587.

Rhudy, J. L., Williams, A. E., McCabe, K. M., Rambo, P. L., \& Russell, J. L. (2006). Emotional modulation of spinal nociception and pain: The impact of predictable noxious stimulation. Pain, 126, 221-233. doi:10.1016/j.pain.2006.06.027

Rhudy, J. L., Williams, A. E., McCabe, K. M., Russell, J. L., \& Maynard, L. J. (2008). Emotional control of nociceptive reactions (ECON): Do affective valence and arousal play a role? Pain, 136, 250-261. doi:10.1016/j.pain.2007.06.031

Sandrini, G., Milanov, I., Willer, J. C., Alfonsi, E., Moglia, A., \& Nappi, G. (1999). Different effect of high doses of naloxone on spinal reflexes in normal subjects and chronic paraplegic patients. Neuroscience Letters, 261, 5-8. doi:10.1016/S0304-3940(98)01000-3

Sandrini, G., Serrao, M., Rossi, P., Romaniello, A., Cruccu, G., \& Willer, J. C. (2005). The lower limb flexion reflex in humans. Progress in Neurobiology, 77, 353-395. doi:10.1016/j.pneurobio.2005.11.003

Schupp, H. T., Cuthbert, B. N., Bradley, M. M., \& Birbaumer, N. (1997). Probe P3 and blinks: Two measures of affective startle modulation. Psychophysiology, 34, 1-6. doi:10.1111/j.1469-8986.1997.tb02409.x 


\section{A. E. WILLIAMS, J. L. RHUDY}

Seligman, M. E. (1970). On the generality of the laws of learning. Psychological Review, 77, 406-418. doi:10.1037/h0029790

Seligman, M. E. (1971). Phobias and preparedness. Behavior Therapy, 2, 307-320. doi:10.1016/S0005-7894(71)80064-3

Vrana, S. R., Spence, E. L., \& Lang, P. J. (1988). The startle probe response: A new measure of emotion? Journal of Abnormal Psychology, 97, 487-491. doi:10.1037/0021-843X.97.4.487

Williams, A. E., \& Rhudy, J. L. (2007). The influence of conditioned fear on human pain thresholds: Does preparedness play a role. The Journal of Pain, 8, 598-606. doi:10.1016/j.jpain.2007.03.004 